\title{
PHENIX results of $\pi^{0}$-hadron correlations
}

\author{
Cheuk-Ping Wong* ${ }^{\dagger}$ \\ Georgia State University \\ E-mail: cwong14@student.gsu.edu
}

\begin{abstract}
High momentum partons lose energy in the QGP that is produced in heavy-ion collisions at RHIC, indicated by the fact that jets from these partons are broadened and less energetic when compared to jets in $p+p$ collisions. This in turn indicates that measurements of jet broadening and energy loss provide important insights to the transport properties of the QGP. In PHENIX, information about the jet width and energy loss is extracted from two-particle angular correlation functions of neutral pions and charged hadrons in $\mathrm{Au}+\mathrm{Au}$ collisions. Background flow is subtracted from the correlation functions of $\mathrm{Au}+\mathrm{Au}$ data with measured flow harmonic coefficients $\left(v_{n}\right)$ from PHENIX. Acoustic Scaling is used to estimate the higher order $(n=3,4)$ vn values, which have not been measured at high $p_{T}$ at RHIC. The comparison of $\mathrm{Au}+\mathrm{Au}$ to $p+p$ results shows jet broadening and away side enhancement in the low associated $p_{T}$ region. Details of the $\mathrm{Au}+\mathrm{Au}$ analysis, the latest results in small systems, and physics implications are discussed.
\end{abstract}

13th International Workshop on High-pT Physics in the RHIC/LHC era

March 19th 2019

Knoxville, Tennessee

${ }^{*}$ Speaker.
${ }^{\dagger}$ For the PHENIX Collaboration

(C) Copyright owned by the author(s) under the terms of the Creative Commons 


\section{Introduction}

Since hard scattered partons lose energy as they traverse the Quark-Gluon Plasma (QGP) created in heavy-ion collisions, such as $\mathrm{Au}+\mathrm{Au}$, the fragmented jets from these partons are modified, meaning they are broader and less energetic. Thus, jet modification relative to $p+p$ jet measurements study can be used to probe the properties of the QGP [1].

\section{2. $\pi^{0}$-Hadron Correlation Analysis}

The soft background dominates over jet signal at RHIC energies. In addition, the limited azimuth and rapidity acceptances of PHENIX make full jet reconstruction even more challenging. Therefore, instead of using full jet reconstruction, two-particle azimuth $(\Delta \phi)$ correlations are measured in PHENIX to probe jets. In PHENIX, the common two-particle correlations species are direct gamma-hadron, and hadron-hadron. Although direct gamma-hadron correlations give access to parent parton initial transverse momentum, direct photons are overwhelmed by large decay photon background from $\pi^{0}$ decays at RHIC. In the case that statistics is crucial, such as $\mathrm{Au}+\mathrm{Au}$ measurement which suffers from subtraction of strong underlying events, $\pi^{0}$-hadron correlation analysis is complementary.

The electromagnetic calorimeters (EMCal) with fine spatial resolution in PHENIX central arm is used to measure decay photon pairs. These decay photon pairs are reconstructed to neutral pions in $4-15 \mathrm{GeV} / \mathrm{c}$. Reconstructed neutral pions within $0.12-0.16 \mathrm{GeV}$ mass window are selected as trigger particles. The drift and pad chamber detectors are also used for charged hadron tracking in 0.5-7 GeV/c with the help of RICH detector to veto electron tracks below $5 \mathrm{GeV} / \mathrm{c}$.

Azimuthal correlation functions are corrected with detector efficiency obtained from PHENIX GEANT3 simulation. Event-mixing is performed to correct $\Delta \phi$ for effects due to PHENIX's central arms geometry. The contribution from combinatorial background $\pi^{0}$ is accounted for in the systematic uncertainty and is estimated using the side-band $\pi^{0}$ in the $0.08-0.11 \mathrm{GeV}$ and $0.165-0.2 \mathrm{GeV}$ mass windows. The underlying event is assumed to have a flat distribution in $p+p$ data. In $\mathrm{Au}+\mathrm{Au}$ data, however, one needs to account for the contribution of flow raised by initial collision geometry. The spatial correlation of the underlying event due to flow can be described as a Fourier series in $\Delta \phi$ as shown in Eq. (2.1)

$$
\frac{d N_{\text {flow }}}{d \Delta \phi}(\Delta \phi)=b_{0}\left[1+\sum v_{n}^{\pi^{0}} v_{n}^{h} \cos (n \cdot \Delta \phi)\right]
$$

where $b_{0}$ is the level of the underlying event obtained from the Absolute Normalization Method [2]. As demonstrated in several experimental observables [3, 4, 5], the coefficients of flow harmonics in the interested $p_{T}$ regions can be factorized to $v_{n}^{\pi^{0}}$ and $v_{n}^{h}$ which are the $n$th order flow harmonic coefficients for $\pi^{0}$ and charged hadron, respectively. While $v_{n}^{h}(n=2,3,4)$ and $v_{2}^{\pi^{0}}$ are interpolated from PHENIX results [6, 7], higher order $v_{n}^{\pi^{0}}$ have not been measured at RHIC in the high momentum region. To estimate $v_{3}^{\pi^{0}}$ and $v_{4}^{\pi^{0}}$, Acoustic Scaling [8] is applied. Acoustic Scaling refers to the ratio of $v_{n}(n=3,4)$ to $\left(v_{2}\right)^{n / 2}$, that is,

$$
g_{n}=\frac{v_{n}}{\left(v_{2}\right)^{n / 2}}
$$


This ratio, denoted as $g_{n}$, is $p_{T}$ independent up to $10 \mathrm{GeV} / \mathrm{c}$ as demonstrated with PHENIX data [8]. Thus, we can obtain charged hadron $g_{n}^{h}$ with existing PHENIX charged hadron $v_{n}^{h}$ results

$$
g_{n}^{h}=\frac{v_{n}^{h}}{\left(v_{2}^{h}\right)^{n / 2}}
$$

and assuming $g_{n}^{h}=g_{n}^{\pi^{0}}$ then apply Eq. (2.2) again to estimate neutral pion $v_{3}^{\pi^{0}}$ and $v_{4}^{\pi^{0}}$ via

$$
v_{n}^{\pi^{0}}=g_{n}^{h}\left(v_{2}^{\pi^{0}}\right)^{n / 2}
$$

\section{Angular Broadening in Au+Au Results}

Results from 2007 PHENIX Au+Au data [5] only removed second order flow harmonics with the assumption that the higher even order flow harmonics are negligible, and the odd order flow harmonics do not exist in heavy-ion collisions. In fact, the third order flow harmonics, which is due to the fluctuations in the initial collision geometry, is non-zero as demonstrated with PHENIX and STAR, and PHOBOS data [7, 9, 10]. This third order triangular flow contributes to the shoulder shape of the away-side of the jet functions. In order to eliminate flow effects and extract jet functions, higher flow harmonics up to the fourth order are subtracted in the present analysis which is using combined 2010 and 2011 PHENIX $200 \mathrm{GeV} \mathrm{Au}+\mathrm{Au}$ data.

Examples of jet functions from combined 2010 and 2011 PHENIX $200 \mathrm{GeV} \mathrm{Au}+\mathrm{Au}$ data in 0-20\% and 20-40\% centrality are shown in Fig. 1 and 2 with Gaussian fits. These new results have subtracted second, third, and fourth order underlying flow harmonics. The away-side jet shape is closer to a Gaussian shape compared to the previous PHENIX results [5] which subtracted the second order flow harmonics only. This improvement is more obvious in the low $p_{T}$ bins where underlying flow is strongest.

The away-side jet width is extracted from the Gaussian fits as shown in Fig. 3 which shows the away-side width decreases as trigger or associate $p_{T}$ increases. Furthermore, the away-side width in the most central events is reduced compared to the old results [5] as the away-side jet functions become more Gaussian with higher order flow harmonics subtracted. However, jet broadening in $\mathrm{Au}+\mathrm{Au}$ results is still observed in the low $p_{T}$ regions compared to $p+p$ results. As the associate $p_{T}$ increases, the $\mathrm{Au}+\mathrm{Au}$ and $p+p$ results converge. Comparing 0-20\% and 20-40\% $\mathrm{Au}+\mathrm{Au}$ results, the centrality dependence is insignificant as the uncertainties of these two centrality ranges overlap.

\section{4. $p_{\text {out }}$ Broadening in Small Systems}

Besides the spatial correlations in the azimuth $\Delta \phi$, one can also study two-particle correlations in momentum space by studying $p_{\text {out }}$ distributions. $p_{\text {out }}$ written in Eq. (4.1) is defined as the transverse component of associated particle $p_{T}^{\text {assoc }}$ with respect to the trigger particle $p_{T}^{\text {trig }}[11,12]$. In Eq. (4.1), $\Delta \phi$ is the azimuth angle between the trigger and associated particles as described in Section 2 .

$$
p_{\text {out }}=p_{T}^{a s s o c} \cdot \sin (\Delta \phi)
$$



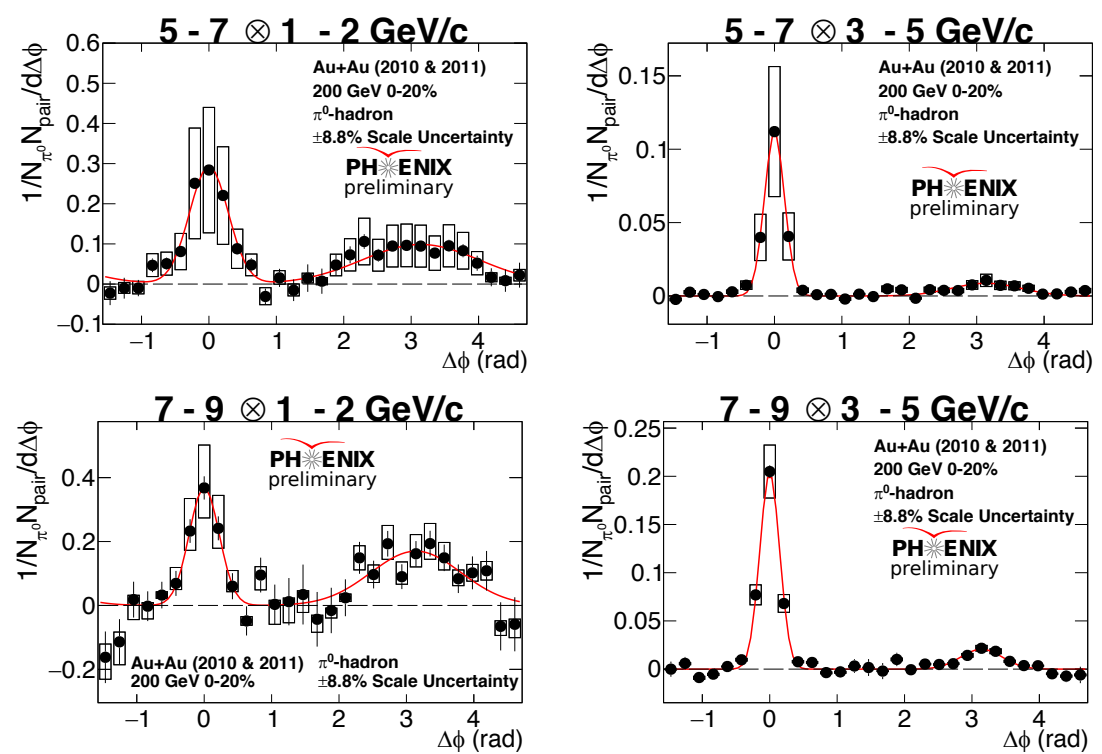

Figure 1: Jet functions with Gaussian fits (red lines) in 0-20\% centrality.
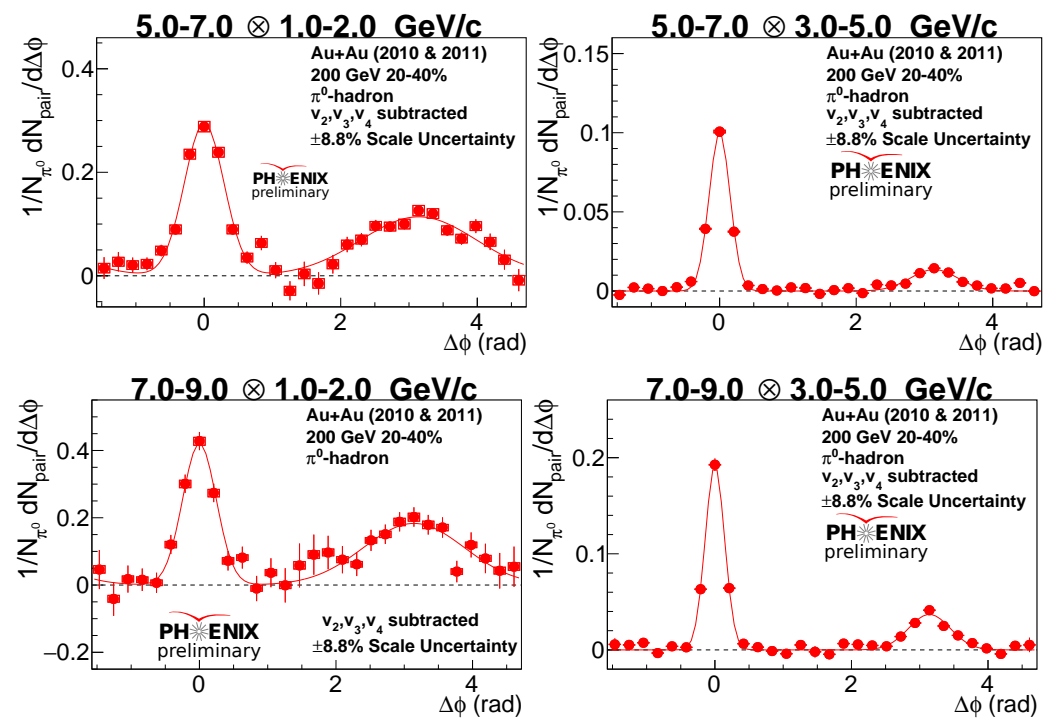

Figure 2: Jet functions with Gaussian fits (red lines) in 20-40\% centrality.

The near-side and away-side $p_{\text {out }}$ distributions from PHENIX $2015 p+p$ and $p+$ Au data are shown in Fig. 4. The color scheme in Fig. 4 separates $p_{\text {out }}$ distributions in different $x_{E}$ ranges. $x_{E}$, defined in Eq. (4.2), is the longitudinal fraction of the $p_{T}^{a s s o c}$ to the $p_{T}^{\text {trig }}$. The $x_{E}$ can serve as an approximation of $z$ which is the ratio of the longitudinal momentum of the away-side jet to the near-side jet in full jet reconstruction [11].

$$
x_{E}=-\frac{\left|p_{T}^{\text {assoc }}\right|}{\left|p_{T}^{\text {trig }}\right|} \cos (\Delta \phi)
$$

Demonstrated in Fig. 4, the away-side $p_{\text {out }}$ distributions are broader than the near-side. This is due to the fact that away-side $p_{\text {out }}$ depends on the initial parton momentum while the near-side $p_{\text {out }}$ does not [12]. Hence, the away-side $p_{\text {out }}$ variation is wider than the near-side $p_{\text {out }}$. 


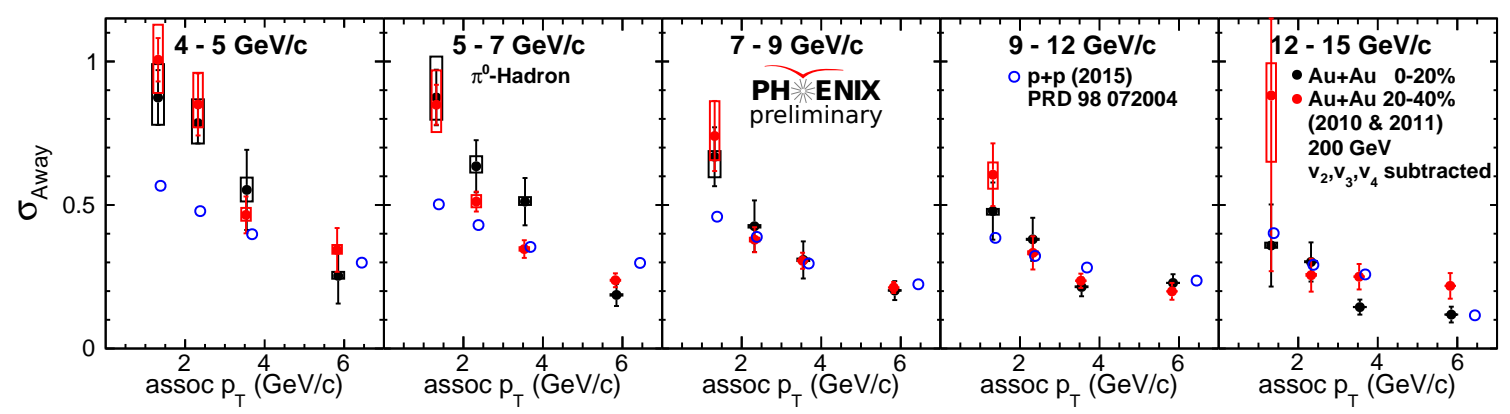

Figure 3: Away-side jet Gaussian width. Results from $200 \mathrm{GeV} \mathrm{Au}+\mathrm{Au}$ in $0-20 \%$ and 20-40\% centralities are shown in black and red dots. Blue circles are PHENIX $2015200 \mathrm{GeV} p+p$ results.
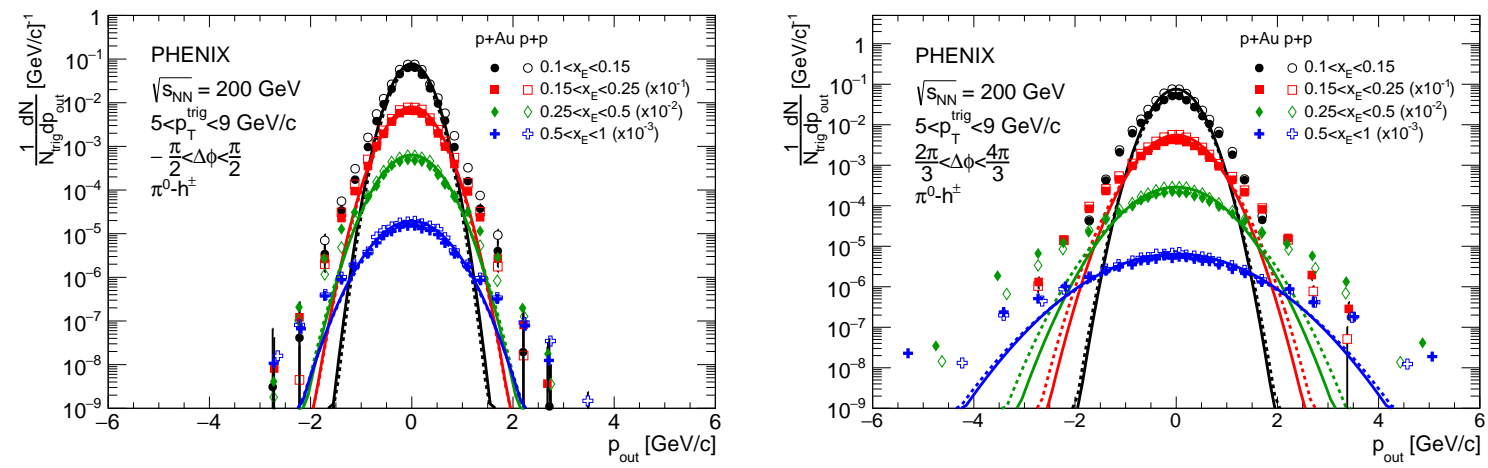

Figure 4: Near-side (left) and away-side (right) $p_{\text {out }}$ distributions in different $x_{E}$ ranges from $200 \mathrm{GeV}$ $p+p$ (solid dots) and $p+\mathrm{Au}$ (open circles) data. The solid and dash lines are the Gaussian fits in the nonperturbative region [12].

The difference of the squared Gaussian width $\left\langle p_{\text {out }}^{2}\right\rangle$ extracted from $p_{\text {out }}$ distributions from $200 \mathrm{GeV} p+p, p+\mathrm{Al}$, and $p+\mathrm{Au}$ data is plotted as a function of $x_{E}$ as shown in Fig. 5. Figure 5 shows no near-side $p_{\text {out }}$ broadening in both $p+\mathrm{Al}$ and $p+\mathrm{Au}$ systems compared to $p+p$ system. In the away side, however, $p_{\text {out }}$ broadening in the $p+\mathrm{Au}$ system is observed, and the broadening enhances in higher $x_{E}$.

Focus on $0.15<x_{E}<0.25$ and $0.25<x_{E}<0.5$, difference of away-side $\left\langle p_{\text {out }}^{2}\right\rangle$ as a function of $N_{\text {coll }}$ is shown in Fig. 6 which demonstrates that $p_{\text {out }}$ is further broadened as $N_{\text {coll }}$ increases. This $N_{\text {coll }}$ dependence could be caused by initial transverse momentum enhancement during multiple hard scattering in the initial collision stage $[13,14]$.

\section{Conclusion}

This presentation gave details in 2010 and $2011 \pi^{0}$-hadron correlation analysis using data from $\mathrm{Au}+\mathrm{Au}$ and small systems collisions measured at PHENIX. The updated $\mathrm{Au}+\mathrm{Au}$ analysis removes the effect of flow harmonics up to the fourth order. The new jet functions in low $p_{T}$ show a more Gaussian shape in the away-side peak than the previous PHENIX results which did not subtract third order flow harmonics. However, the away-side jet broadening still remains in the low associated $p_{T}$ region compared to $p+p$ results. As the associated $p_{T}$ rises, the away-side widths from $\mathrm{Au}+\mathrm{Au}$ and $p+p$ results converge. There is no centrality dependence observed in away-side 


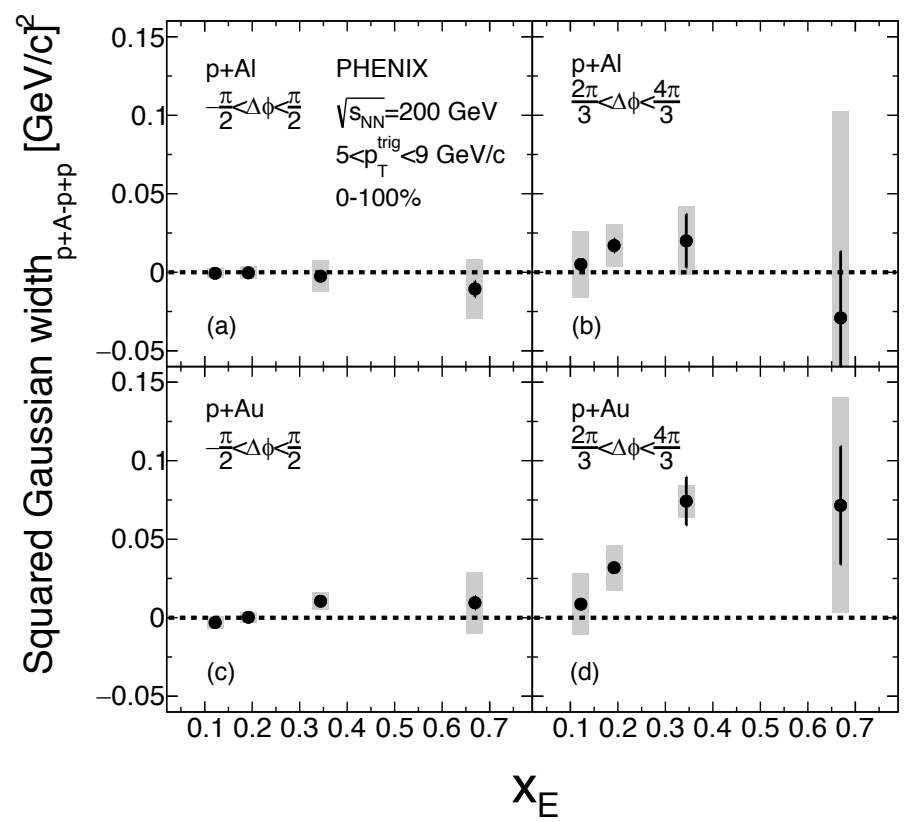

Figure 5: Difference in $\left\langle p_{\text {out }}^{2}\right\rangle$ as a function of $x_{E}$. Top row is $200 \mathrm{GeV} p+\mathrm{Al}$ results in full centrality range, bottom row is $200 \mathrm{GeV} p+\mathrm{Au}$ results in full centrality range. Left and right columns are difference in $\left\langle p_{\text {out }}^{2}\right\rangle$ from near and away side measurements, respectively [12].

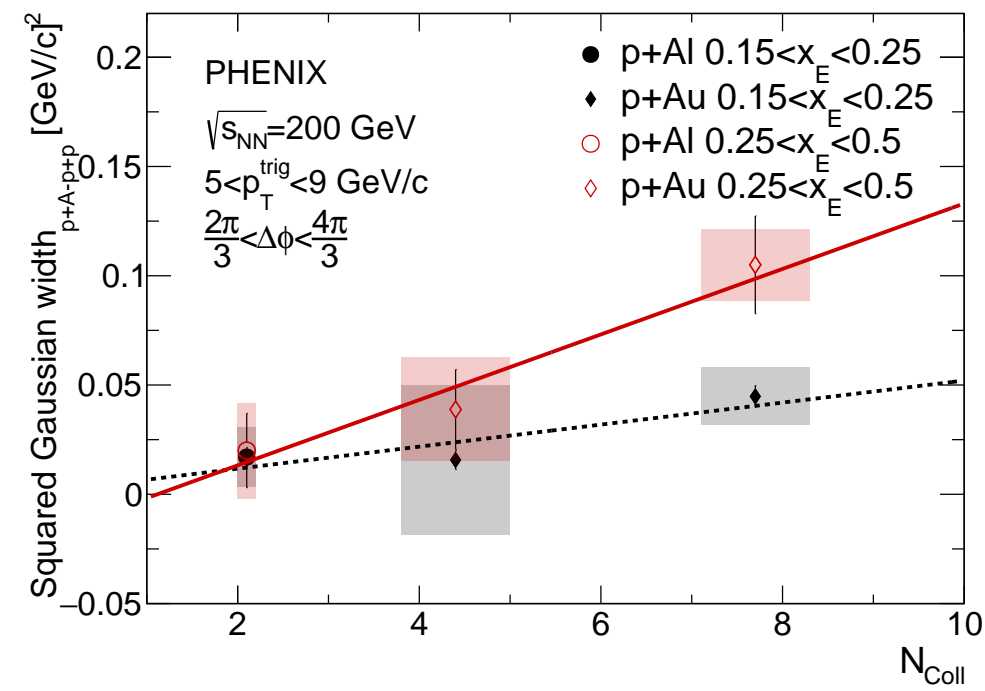

Figure 6: Difference in $\left\langle p_{\text {out }}^{2}\right\rangle$ as a function of $N_{\text {coll }}$. Results in $0.15-0.25$ and $0.25-0.5 x_{E}$ bins are drawn in black and red, respectively. $p+\mathrm{Al}$ results are drawn in circle or dot markers, while open and solid diamond markers represent $p+\mathrm{Au}$ results. The red and balck lines are linear fits to the data [12].

widths from the comparison of $0-20 \%$ to $20-40 \%$ centrality ranges as the uncertainties in these ranges overlap.

Jet broadening in small systems is also shown in momentum space in the study of $p_{\text {out }}$. While modification of the near-side $p_{\text {out }}$ is not observed, away-side $p_{\text {out }}$ broadening is shown in $p+\mathrm{Au}$ data compared to $p+p$. Indication of correlation between away-side $p_{\text {out }}$ broadening and $N_{\text {coll }}$ is found. These observations could be due to boost of initial transverse momentum in the beginning 
of collision. These results, which give insight to cold nuclear matter effects, are also important for understanding hot nuclear matter effect in the study of QGP medium in heavier systems.

\section{Acknowledgments}

This work was supported by the National Science Foundation [grant number 1714802].

\section{References}

[1] M. Connors, C. Nattrass, R. Reed, S. Salur, Jet measurements in heavy ion physics, Rev. Mod. Phys. 90 (2018) 025005. doi:10.1103/RevModPhys.90.025005.

[2] A. Sickles, M. P. McCumber, A. Adare, Extraction of correlated jet pair signals in relativistic heavy ion collisions, Phys. Rev. C 81 (2010) 014908. doi:10.1103/PhysRevC.81.014908.

[3] J. Adam, et al., Jet-like correlations with neutral pion triggers in pp and central pb-pb collisions at 2.76 TeV, Physics Letters B 763 (2016) 238 - 250. doi:10.1016/j.physletb.2016.10.048.

[4] L. Adamczyk, et al., Jet-like correlations with direct-photon and neutral-pion triggers at $\sqrt{s_{N N}}=200$ GeV, Physics Letters B 760 (2016) 689 - 696. doi:10.1016/j.physletb.2016.07.046.

[5] A. Adare, et al., Transition in yield and azimuthal shape modification in dihadron correlations in relativistic heavy ion collisions, Phys. Rev. Lett. 104 (2010) 252301. doi:10.1103/PhysRevLett.104.252301.

[6] A. Adare, et al., Measurement of two-particle correlations with respect to second and third-order event planes in $\mathrm{Au}+\mathrm{Au}$ collisions at $\sqrt{s_{N N}}=200 \mathrm{GeV}$, Phys. Rev. C 99 (2019) 054903. doi:10.1103/PhysRevC.99.054903.

[7] A. Adare, et al., Measurements of higher-order flow harmonics in au+au collisions at $\sqrt{s_{N N}}=200$ GeV, Phys. Rev. Lett. 107 (2011) 252301. doi:10.1103/PhysRevLett.107.252301.

[8] R. A. Lacey, et al., Scaling of the higher-order flow harmonics: implications for initial-eccentricity models and the.

[9] L. Adamczyk, et al., Third harmonic flow of charged particles in au + au collisions at $\sqrt{s_{N N}}=200$ gev, Phys. Rev. C 88 (2013) 014904. doi:10.1103/PhysRevC.88.014904.

[10] B. Alver, G. Roland, Collision-geometry fluctuations and triangular flow in heavy-ion collisions, Phys. Rev. C 81 (2010) 054905. doi:10.1103/PhysRevC.81.054905.

[11] C. Aidala, et al., Nonperturbative transverse-momentum-dependent effects in dihadron and direct photon-hadron angular correlations in $p+p$ collisions at $\sqrt{s}=200 \mathrm{GeV}$, Phys. Rev. D 98 (2018) 072004. doi:10.1103/PhysRevD.98.072004.

[12] C. Aidala, et al., Nonperturbative-transverse-momentum broadening in dihadron angular correlations in $\sqrt{s_{N N}}=200 \mathrm{GeV}$ proton-nucleus collisions, Phys. Rev. C 99 (2019) 044912. doi:10.1103/PhysRevC.99.044912.

[13] J. W. Cronin, et al., Production of hadrons at large transverse momentum at 200, 300, and 400 gev, Phys. Rev. D 11 (1975) 3105-3123. doi:10.1103/PhysRevD.11.3105.

[14] M. Corcoran, et al., Evidence for multiple scattering of high energy partons in nuclei, Physics Letters B 259 (1) (1991) 209 - 215. doi:https://doi.org/10.1016/0370-2693(91)90160-R. 